\title{
The role of complete blood count parameters in distinguishing complicated and uncomplicated appendicitis
}

\author{
(1) Talha Sarıgöz ${ }^{1}$, (1) Uğur Aydemir ${ }^{2}$ \\ ${ }^{1}$ Kayseri City Hospital, Clinic of General Surgery, Kayseri, Turkey \\ ${ }^{2}$ Aksaray State Hospital, Clinic of General Surgery, Aksaray, Turkey
}

Date submitted:

12.12.2020

Date accepted:

13.01.2021

Online publication date:

15.09.2021

\section{Corresponding Author:}

Talha Sarıgöz, M.D., Kayseri City

Hospital, Clinic of General Surgery,

Kayseri, Turkey

sarigozt.md@gmail.com

ORCID:

orcid.org/ 0000-0001-6573-6519

Keywords: Appendicitis, complicated appendicitis, white blood cell count, neutrophil to lymphocyte ratio

\begin{abstract}
Aims: Acute appendicitis is a prevalent disease. In recent years, conservative treatment of acute uncomplicated appendicitis has been popular but complicated disease requires surgery. This study was conducted to search whether white blood cell (WBC) count, mean platelet volume (MPV), red blood cell distribution width (RDW), and neutrophil-to-lymphocyte ratio (NLR) might be useful to distinguish complicated and uncomplicated appendicitis.

Methods: In this single-center retrospective study, we evaluated patients who underwent open appendectomy between January 2013 and December 2015. Then, the subjects were divided into two groups according to surgical exploration and pathological examination: complicated appendicitis and uncomplicated appendicitis. Complicated cases were classified into 3 subgroups: 1) appendix perforation, 2) plastron appendicitis, and 3) necrosis of appendix. WBC, MPV, RDW, and NLR values were compared in all groups.
\end{abstract}

Results: A total of 1,219 patients were analyzed. Complicated appendicitis was more common in male patients $(n=71,9.6 \%)$. The median WBC $\left(16.1 \times 10^{9} / L\right)$ and NLR $(7.9 n / \mu L)$ were significantly higher in complicated appendicitis compared to uncomplicated disease ( $p<0.001$, for both). The cut-off value for WBC and NLR to distinguish a complicated disease was $14.6 \times 10^{9} / \mathrm{L}$ and 6.2 $\mathrm{n} / \mu \mathrm{L}$, respectively. MPV and RDW were not useful.

Conclusions: WBC and NLR may be helpful in distinguishing between complicated and uncomplicated appendiceal disease. However, we observe no significant differences in RDW and MPV levels in patients with complicated appendicitis.

\section{Introduction}

Acute appendicitis (AA) is a prevalent disease that can be lifethreating if left untreated. There have been advances in imaging techniques recently, which facilitated the hand of physicians, but they are not widely available and require expertise to interpret. This makes diagnosis of appendicitis challenging for physicians working in rural regions. Complete blood count (CBC) is still the first line of test for a patient with abdominal pain in emergency departments (1). It is an easy and quick test to evaluate hematologic parameters which can be changed due to infection, hemorrhage and genetic disorders.

Systemic inflammatory response is generally associated with white blood cell (WBC) count (2). However, WBC has no role in differentiating simple and complicated appendiceal disease.
In addition to WBC count, there are several other markers that can be used as a sign of inflammatory conditions. For instance, neutrophil-to-lymphocyte ratio (NLR) is an indicator of subclinical inflammation (3). Also, red blood cell distribution width (RDW) has been reported to be in relation to infection (4). Furthermore, platelets play a crucial role in inflammation and their size can change as a result of inflammatory conditions. Therefore, mean platelet volume (MPV), which is a measurement of the average size of platelet in the blood, may have diagnostic potential in various diseases $(5,6)$.

In this study, we aimed to identify whether there was a relationship between the severity of $A A$ and WBC, NLR, RDW, and MPV at the time of presentation to the emergency department. 


\section{Methods}

This retrospective study was conducted at Kayseri City Hospital after obtaining approval from Erciyes University Scientific Research Ethics Committee (2017/599).

Database was searched retrospectively for patients with discharge diagnosis of $\mathrm{AA}$, who underwent open appendectomy between January 2013 and December 2015, using keywords "acute appendicitis". The initial search delivered 1,926 patients. Records lacking pathological examination reports, preoperative laboratory evaluation, clinical and demographic parameters were excluded. Also, patients younger than 18 years of age, having hematologic disease and diagnosed with incidental appendiceal malignancy were left out of the study. In total, 1,219 records which fulfilled all the criteria were included in the study. The following variables were extracted for each patient: age, gender, admission CBC, surgical findings and pathological examination report.

The subjects were divided into two groups according to surgical exploration and pathological examination results, as complicated appendicitis and uncomplicated appendicitis. Complicated cases involved 3 subgroups: 1) appendix perforation 2) plastron appendicitis 3) necrosis of appendix. The study population was also evaluated in terms of how age affected CBC parameters. They were divided into additional two groups according to age over 65 years and under. WBC, NLR, RDW and MPV values were assessed among the groups.

\section{Statistical Analysis}

Statistical analysis was performed on Statistical Package for the Social Sciences (SPSS) 23.0 software package (SPSS, Inc., Chicago, Illinois). Categorical data were expressed as number (percentage) and continuous data as median interquartile range (IQR) $25^{\text {th }}-75^{\text {th }}$ percentile. For statistical comparison between the groups, the chi-square test for categorical data and the Mann-Whitney $U$ test for continuous data were used. Also, receiver operating characteristics (ROC) analysis was used to investigate the status of $\mathrm{CBC}$ parameters in diagnosis of complicated appendicitis. Statistical significance was accepted at 0.05 .

\section{Results}

A total of 1,219 patients who underwent open appendectomy were included in the analysis. While the majority of the patients were male $(60.5 \%)$, the median age was 30 (IQR: 22-42) years. While $1,108(90.9 \%)$ patients had uncomplicated appendicitis, $79(6.5 \%)$ had perforated, $11(0.9 \%)$ had necrotic and $21(1.7 \%)$ had plastron appendicitis, which were confirmed with pathological examination. Whilst patients with plastron appendicitis were older, subjects having necrosis of the appendix were the youngest among the groups $(p<0.001)$. The male patients presented with slightly higher rates of perforation and the female patients had slightly higher rates of appendix necrosis. The median appendix length was $6.5 \mathrm{~mm}$ (IQR: 5-8) according to the pathological examination.

When all of the patients with $A A$ were evaluated, the median value of WBC was $13.5 \times 10^{\circ} / \mathrm{L}$. However, patients older than 65 years of age had mildly lower WBC counts but the difference between those and younger patients was not statistically significant $(p=0.115)$. On the other hand, the patients presenting with necrosis of the appendix had the highest WBC counts and there was statistically significant difference among the groups in terms of WBC $(p<0.001)$. In addition, uncomplicated appendicitis cases had the lowest WBC counts (median 13.4×10\%/L).

The median MPV value was $13.3 \mathrm{fL}$ (IQR: 10.2-14.7) when all of the patients were evaluated. Including patients older than 65 years of age and younger $(p=0.071)$, there was not statistically significant difference when MPV values were compared among the groups $(p=0.060)$. However, MPV values of the patients with plastron appendicitis or appendix necrosis were slightly lower than uncomplicated group and patients with appendix perforation. When the RDW values were compared, there was also not statistically significant difference among the groups $(p=0.129)$.

In addition to WBC, MPV and RDW, NLR was also evaluated. For patients $<65$ and $\geq 65$, there was no significant difference in terms of NLR values ( $p=0.182)$, but NLR was significantly different between complicated and uncomplicated cases $(p<0.001)$. The highest NLR values were observed in patients with necrosis of appendicitis (median, $9.8 \mathrm{n} / \mu \mathrm{L}$ ). The uncomplicated cases had the lowest median NLR value (median, $5.1 \mathrm{n} / \mu \mathrm{L}$ ) among the groups (Table 1).

Diagnostic potential of $\mathrm{CBC}$ parameters was evaluated by the ROC analysis. WBC and NLR values were found to be meaningful in discriminating complicated disease from uncomplicated $(p<0.001)$. For WBC, the cut-off value for the diagnosis of complicated appendicitis was $14.6 \times 10^{9} / \mathrm{L}$ with sensitivity of $64.1 \%$ and, for NLR, it was $6.2 \mathrm{n} / \mu \mathrm{L}$ with a sensitivity of $60.3 \%$. The area under the ROC curve was 0.687 for WBC and 0.670 for NLR. WBC was a more accurate predictor of complicated appendicitis (Figure 1, Table 2).

\section{Discussion}

The study results indicated that while majority of the patients were male, patients tended to present with appendix perforation with increasing age. From the $\mathrm{CBC}$ parameters, only WBC and NLR were found to be associated with disease severity. The highest WBC and NLR were observed in patients with appendix necrosis, followed by perforation and plastron disease. Higher WBC values than $14.6 \times 10^{\circ} / \mathrm{L}$ were indicative of complicated disease with a sensitivity of $64.1 \%$. For NLR, the cut-off for complicated disease was $6.2 \mathrm{n} / \mu \mathrm{L}$ with a sensitivity of $60.3 \%$. MPV and RDW were not informative of appendiceal disease. In this study, $\mathrm{CBC}$ had limited role in distinguishing complicated appendicitis from uncomplicated. 


\begin{tabular}{|c|c|c|}
\hline \multicolumn{2}{|l|}{ Values } & $p$ value \\
\hline Age, years, mean (range) & $30(22-42)$ & \multirow{5}{*}{$<0.001$} \\
\hline Uncomplicated & $29(22-41)^{a}$ & \\
\hline Perforated & $31(20-44)^{b}$ & \\
\hline Plastron & $43(37-61)^{c}$ & \\
\hline Necrosis & $20(18-25)^{d}$ & \\
\hline \multicolumn{2}{|l|}{ Gender, n (\%) } & \multirow{11}{*}{-} \\
\hline Male & $738(60.5)$ & \\
\hline Uncomplicated & $667(90.4)$ & \\
\hline Perforated & $52(7)$ & \\
\hline Plastron & $13(1.8)$ & \\
\hline Necrosis & $6(0.8)$ & \\
\hline Female & $481(39.5)$ & \\
\hline Uncomplicated & $441(91.6)$ & \\
\hline Perforated & $27(5.6)$ & \\
\hline Plastron & $8(1.7)$ & \\
\hline Necrosis & $5(1)$ & \\
\hline Appendix length, mm & $6.5(5-8)$ & - \\
\hline WBC, $10^{9} / \mathrm{L}$ & $13.5(11-16)$ & \\
\hline$<65$ & $13.5(11.1-16.1)$ & 0.115 \\
\hline$\geq 65$ & $13(9.9-15.1)$ & \\
\hline Uncomplicated & $13.4(10.9-15.7)^{\mathrm{a}}$ & \\
\hline Perforated & $16.1(13.3-20)^{\mathrm{b}}$ & $<0.001$ \\
\hline Plastron & $15.1(12.6-16.6)^{c}$ & \\
\hline Necrosis & $25.2(23.3-26.3)^{d}$ & \\
\hline MPV, fL & $13.3(10.2-14.7)$ & \\
\hline$<65$ & $13.3(10.2-14.6)$ & 0.071 \\
\hline$\geq 65$ & $14(10.8-15.5)$ & \\
\hline Uncomplicated & $13.3(10.5-14.7)^{\mathrm{a}}$ & \\
\hline Perforated & $13.5(12-14.9)^{\mathrm{a}}$ & \\
\hline Plastron & $11.6(8-13.5)^{a}$ & 0.060 \\
\hline Necrosis & $12.9(12-14.9)^{\mathrm{a}}$ & \\
\hline RDW, \% & $8.9(7.8-25.6)$ & \\
\hline$<65$ & $8.9(7.8-30)$ & 0.945 \\
\hline$\geq 65$ & $9(7.4-26)$ & \\
\hline Uncomplicated & $8.9(7.7-12.4)^{a}$ & \\
\hline Perforated & $9(7.8-11.1)^{a}$ & 0129 \\
\hline Plastron & $11(7.9-45)^{\mathrm{a}}$ & 0.129 \\
\hline Necrosis & $10.4(9.8-42)^{a}$ & \\
\hline NLR, $n / \mu L$ & $5.3(3.4-8.7)$ & \\
\hline$<65$ & $5.3(3.4-8.6)$ & 0.182 \\
\hline$\geq 65$ & $5.2(3.6-12.1)$ & \\
\hline Uncomplicated & $5.1(3.3-8.3)^{a}$ & \\
\hline Perforated & $8.4(5.3-12.7)^{\mathrm{b}}$ & $<0.001$ \\
\hline Plastron & $6(4.1-10.8)^{c}$ & \\
\hline Necrosis & $9.8(4.4-16.4)^{d}$ & \\
\hline
\end{tabular}

The definition of complicated appendicitis is usually used for appendicitis with signs of perforation, phlegmon or abscess (7). According to the study by Perez and Allen (7), complicated appendicitis mostly occurs in males. In our study, the percentage

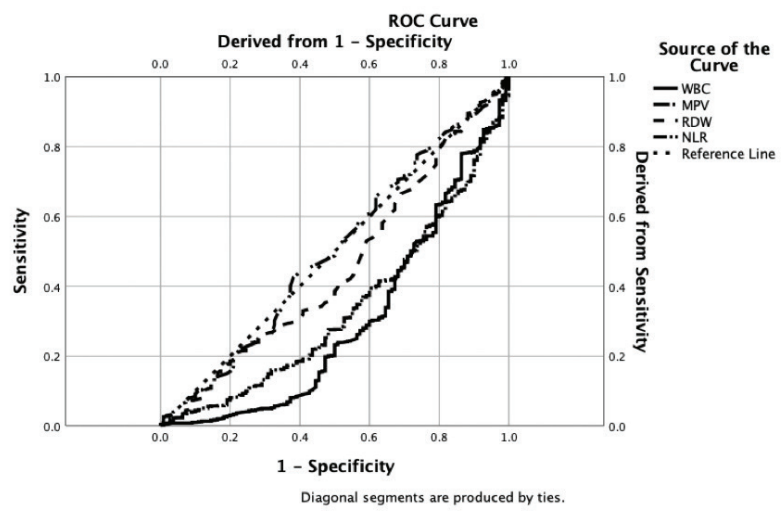

Figure 1. Graphical representation of the receiver operating characteristic curve

ROC: Receiver operating characteristics, WBC: White blood cell, MPV: Mean platelet volume, NLR: Neutrophil-to-lymphocyte ratio, RDW: Red blood cell distribution width

\begin{tabular}{|c|c|c|c|c|c|}
\hline \multirow{2}{*}{ Variable } & \multirow{2}{*}{$\begin{array}{l}\text { Cut-off } \\
\text { value }\end{array}$} & \multirow{2}{*}{$\begin{array}{l}\text { Area } \\
\text { under } \\
\text { curve }\end{array}$} & \multirow{2}{*}{$p$ value } & \multicolumn{2}{|c|}{$\begin{array}{l}95 \% \text { confidence } \\
\text { interval }\end{array}$} \\
\hline & & & & $\begin{array}{l}\text { Lower } \\
\text { bound }\end{array}$ & $\begin{array}{l}\text { Upper } \\
\text { bound }\end{array}$ \\
\hline WBC, $10^{9} / \mathrm{L}$ & 14.6 & 0.687 & $<0.001$ & 0.618 & 0.755 \\
\hline MPV, fL & - & 0.547 & 0.765 & 0.483 & 0.611 \\
\hline RDW, \% & - & 0.510 & 0.163 & 0.444 & 0.576 \\
\hline$N L R, n / \mu L$ & 6.2 & 0.670 & $<0.001$ & 0.608 & 0.732 \\
\hline
\end{tabular}

of complication in males was $9.6 \%$ while it was $8.3 \%$ in females. We also observed that subjects with either plastron or perforation were significantly older than patients with uncomplicated appendicitis. However, cases with appendix necrosis were significantly younger than uncomplicated cases. It is hard to find a similar result in the literature because complicated cases were mostly evaluated as a whole. In their study, Eddama et al. (8) reported that patients with complicated appendicitis were significantly older than patients with uncomplicated appendicitis, but they did not perform subgroup analysis similar to this study. The results of this study regarding necrosis may be interpreted as younger patients tend to delay hospital submission, the appendiceal disease may progress.

It would be ideal if appendiceal disease had a unique marker. In this regard, multiple studies have looked at varied markers. However, there is no general consensus that would have been reached declaring one marker as the gold standard. WBC remains the most common marker used in AA diagnosis. According to this study results, WBC values were significantly different among the groups. The highest values were observed in cases with necrosis followed by appendix perforation and 
plastron disease. In the systematic review by Acharya et al. (9), the pooled sensitivity of WBC count was 0.79 and the specificity was 0.55 . For the diagnosis of perforated appendicitis, the sensitivity was 0.70 and the specificity was 0.49 . In this work up, in patients older than 65 years of age, relatively low levels of WBC count were observed. However, in $83.5 \%$ of the patients, WBC count was higher than then the upper limit of normal, which is $10 \times 10^{9} / \mathrm{L}$ for the institution. In the elderly, low levels of WBC should not dissuade physicians from AA suspicion. However, there are also conflicting papers in the literature. For instance, Tind et al. (10) concluded that leucocyte counts did not influence clinical decision-making. We believe, when combined with anamnesis and physical examination, elevated levels of WBC are highly accurate in AA diagnosis. The cut-off value for WBC in diagnosis of complicated appendicitis was $14.6 \times 10^{9} / \mathrm{L}$. The sensitivity was $64.1 \%$, which is acceptable considering not only perforation but also plastron and necrotic appendiceal disease was included in the complicated group.

To increase the accuracy of AA diagnosis, researchers have used many laboratory parameters. One of them is MPV because platelets play a crucial role in inflammation and biomarkers such as MPV are in relation to platelet morphology. In the study of Daldal and Dagmura (11), they found elevated MPV values in patients with appendix diameter of $<6 \mathrm{~mm}$. Considering the that the median diameter of appendix was $6.5 \mathrm{~mm}$ in our study, we could not find significant difference among the groups in relation to MPV. Bozkurt et al. (12) and Dinc et al. (13) also suggested that there was an association between AA and MPV. In the work of Tanrikulu et al. (14), they found significantly lower levels of MPV in patients with AA than healthy subjects, but reported sensitivity was $45 \%$ and specificity $89.2 \%$. However, WBC was $76.2 \%$ sensitive and $90.5 \%$ specific in diagnosis of AA. It seems MPV has limited role in AA diagnosis.

It has been reported in the recent years that high RDW values could be a new prognostic indicator that may reflect an underlying inflammatory condition (15). Additionally, it has been reported to be related to outcome in cases of infection, especially sepsis (3). However, in this work-up, we could not demonstrate significant difference among the groups in terms of RDW. In a study by Boshnak et al. (1), they also indicated that RDW was not useful in AA diagnosis.

In the study by Daldal and Dagmura (11), they also reported that NLR was an important parameter if appendix diameter was $>6 \mathrm{~mm}$. In this study, we found similar results to their study. NLR was significantly different between complicated and uncomplicated cases. In their meta-analysis, Hajibandeh et al. (3) observed similar findings for NLR. They reported that NLR of $8.8 \mathrm{n} / \mu \mathrm{L}$ was cut-off for complicated appendicitis (sensitivity $76.92 \%$, specificity $100 \%$ ). However, we only observed higher values in patients with appendix necrosis. Our results for plastron disease and appendix perforation did not consist with their results. Also, we found the cut-off for complicated appendicitis as $6.2 \mathrm{n} / \mu \mathrm{L}$, which was relatively lower than that Hajibandeh et al. (3) reported.

However, there are limitations to this study. First, due to retrospective nature of the study, there were not clear data about comorbidities on the database. Therefore, impact of comorbidities could not be evaluated among the groups. But also, considering that the subjects were relatively young, significant impact of comorbidities is not expected. Furthermore, it is within possibility that some patients may have been admitted to other hospitals earlier and delay in submission might have affected laboratory parameters.

\section{Conclusion}

In conclusion, determining the severity of appendicitis is considered important because a complicated disease can require a longer hospitalization period. In the light of the literature and present results, WBC may be the most important parameter in the diagnosis of AA. NLR also seems to be increased in patients with complicated appendicitis. This study did not find any difference in the level of RDW and MPV in patients with complicated appendicitis.

\section{Ethics}

Ethics Committee Approval: This study was performed after obtaining approval from Erciyes University Scientific Research Ethics Committee (numbered: 2017/599).

Informed Consent: Retrospective study.

Peer-review: Externally peer-reviewed.

\section{Authorship Contributions}

Surgical and Medical Practices: T.S., U.A., Concept: T.S., U.A., Design: T.S., U.A., Data Collection or Processing: T.S., U.A., Analysis or Interpretation: T.S., U.A., Literature Search: T.S., U.A., Writing: T.S., U.A.

Conflict of Interest: No conflict of interest was declared by the authors.

Financial Disclosure: The authors declared that this study received no financial support.

\section{References}

1. Boshnak N, Boshnaq M, Elgohary $\mathrm{H}$. Evaluation of Platelet Indices and Red Cell Distribution Width as New Biomarkers for the Diagnosis of Acute Appendicitis. J Invest Surg. 2018;31:121-129.

2. Zahorec R. Ratio of neutrophil to lymphocyte counts--rapid and simple parameter of systemic inflammation and stress in critically ill. Bratisl Lek Listy. 2001;102:5-14.

3. Hajibandeh S, Hajibandeh S, Hobbs N, Mansour M. Neutrophil-to-lymphocyte ratio predicts acute appendicitis and distinguishes between complicated and uncomplicated 
appendicitis: A systematic review and meta-analysis. Am J Surg. 2020;219:154-163.

4. Nishizaki $Y$, Daida H. Red blood cell distribution width for heart failure. Intern Med. 2013;52:417.

5. Thachil J. Platelets in Inflammatory Disorders: A Pathophysiological and Clinical Perspective. Semin Thromb Hemost. 2015;41:572-581.

6. Bhangu A, Søreide K, Di Saverio S, Assarsson JH, Drake FT. Acute appendicitis: modern understanding of pathogenesis, diagnosis, and management. Lancet. 2015;386:1278-1287.

7. Perez KS, Allen SR. Complicated appendicitis and considerations for interval appendectomy. JAAPA. 2018;31:35-41.

8. Eddama M, Fragkos KC, Renshaw S, et al. Logistic regression model to predict acute uncomplicated and complicated appendicitis. Ann R Coll Surg Engl. 2019;101:107-118.

9. Acharya A, Markar SR, Ni M, Hanna GB. Biomarkers of acute appendicitis: systematic review and cost-benefit trade-off analysis. Surg Endosc. 2017;31:1022-1031.
10. Tind S, Lassen AT, Zimmerman-Nielsen E, Qvist N. C-reactive protein and white blood cell count do not improve clinical decision-making in acute appendicitis. Dan Med J. 2015;62:A5167.

11. Daldal E, Dagmura H. The Correlation between Complete Blood Count Parameters and Appendix Diameter for the Diagnosis of Acute Appendicitis. Healthcare (Basel). 2020;8:39.

12. Bozkurt S, Köse A, Erdogan S, et al. MPV and other inflammatory markers in diagnosing acute appendicitis. J Pak Med Assoc. 2015;65:637-641.

13. Dinc T, Senol K, Yildiz B, Kayilioglu I, Sozen I, Coskun F. Association between red cell distribution width and mean platelet volume with appendicitis: a myth or a fact? Bratisl Lek Listy. 2015;116:499-501.

14. Tanrikulu CS, Tanrikulu Y, Sabuncuoglu MZ, Karamercan MA, Akkapulu N, Coskun F. Mean platelet volume and red cell distribution width as a diagnostic marker in acute appendicitis. Iran Red Crescent Med J. 2014;16:e10211.

15. Hong N, Oh J, Kang SM, et al. Red blood cell distribution width predicts early mortality in patients with acute dyspnea. Clin Chim Acta. 2012;413:992-997. 